\title{
Estimates of diffusion due to long-range beam-beam collisions
}

\author{
Y. Papaphilippou* \\ Brookhaven National Laboratory, Upton, New York 11973 \\ F. Zimmermann \\ CERN, CH-1211 Geneva 23, Switzerland \\ (Received 15 February 2002; published 22 July 2002)
}

\begin{abstract}
Weak-strong tracking simulations for the Large Hadron Collider have shown that long-range beambeam collisions give rise to a well-defined diffusive aperture beyond which particles are lost quickly. In order to derive analytical estimates of this stability boundary, we use leading order perturbation theory and the Chirikov resonance overlap criterion applied to a simplified model with a 2-dimensional transverse phase space. In addition, a Fokker-Plank-type diffusion coefficient is calculated through the nonlinear action kicks imparted by the long-range beam-beam force. The analytical results are compared with the tracking data.
\end{abstract}

DOI: 10.1103/PhysRevSTAB.5.074001

\section{INTRODUCTION}

In a colliding-beam storage ring, one of the largest perturbations affecting the motion of beam particles is the collision with the opposing beam. This interaction occurs, unavoidably, in the form of head-on collisions between bunches of the two beams at designated interaction points (IPs) with minimum beta function. Many past studies for colliding proton beams have shown that simulations of head-on collisions can only reproduce the experimental data if a betatron-tune modulation of the order of $10^{-4}$ is included (see, for example, [1-3]). In addition, a transverse offset between the closed orbits of the two colliding beams at the head-on collision point has strongly enhanced the diffusion and particle losses, both in simulations and in experiments [3].

Future colliders employ long trains of closely spaced bunches, and individual bunches encounter many others of the opposing beam at various long-range (or "parasitic") collision points, where the beams are not fully separated. In general, the effect of the long-range collisions depends on the ratio of the beam separation to the local rms beam size and on the total number of long-range collision points. In the case of the Large Hadron Collider (LHC), a 7-TeV double-ring proton collider presently under construction at CERN, the long-range collisions occur in the vicinity of each main head-on IP, before the beams are fully separated into two disjunct beam pipes. Therefore, in the LHC the effective strength of the long-range collisions depends on the ratio of the beam crossing angle to the rms beam divergence at the main IPs. On either side of the two LHC main collision points, a beam encounters about 15 parasitic collisions with an approximate average separation between the closed orbits of the two beams of $9.5 \mathrm{rms}$ beam sizes (see Table I).

*Present address: ESRF, BP 220, Grenoble Cedex, France.
PACS numbers: 29.27.Bd, 05.45.-a

Simulations predict that the long-range collisions in hadron colliders give rise to a well-defined border of stability at an amplitude which we call the "diffusive aperture" [4-6]. As an example, Fig. 1 shows the change of the action variance $(\Delta J)_{x, y}^{2}$ per turn, normalized to the square of the transverse emittance $\varepsilon_{x, y}$, as computed by beam-beam simulations which consider the particle motion in a 4-dimensional transverse phase space for a model with two IPs and parameters similar to those of the LHC [5]. The diffusive aperture is insensitive to the presence of the head-on collision (filled circles with dark blue curve), and only marginally affected by the nonlinear field errors in the final-triplet quadrupoles (squares with green curve), or by a small additional tune ripple (empty circles with pink curve). The diffusive aperture with long-range collisions is equally insensitive to transverse closed-orbit offsets between the two beams at the head-on collision points [5]. Thus, previous studies for head-on collisions are not directly applicable, and a better understanding of the role of the long-range interaction is called for.

In this article, we first present a few simulation results obtained by applying either the full beam-beam force or a $1 / r^{\prime}$ approximation. We then derive analytical estimates of the diffusive aperture induced by the long-range collisions.

TABLE I. Simulation parameters.

\begin{tabular}{lcc}
\hline \hline \multicolumn{1}{c}{ Variable } & Symbol & Value \\
\hline Beam energy & $E$ & $7 \mathrm{TeV}$ \\
Particle species & $\ldots$ & protons \\
Full crossing angle & $\theta_{c}$ & $300 \mu \mathrm{rad}$ \\
rms beam divergence & $\sigma_{x}^{\prime}$ & $31.7 \mu \mathrm{rad}$ \\
rms beam size & $\sigma_{x}$ & $15.9 \mu \mathrm{m}$ \\
Normalized transv. & & $3.75 \mu \mathrm{m}$ \\
$\quad$ rms emittance & $\gamma \varepsilon$ & $0.5 \mathrm{~m}$ \\
IP beta function & $\beta^{*}$ & $\left(1 \times 10^{11}-2 \times 10^{12}\right)$ \\
Bunch charge & $N_{b}$ & 0.31 \\
Betatron tune & $Q_{0}$ & \\
\hline \hline
\end{tabular}




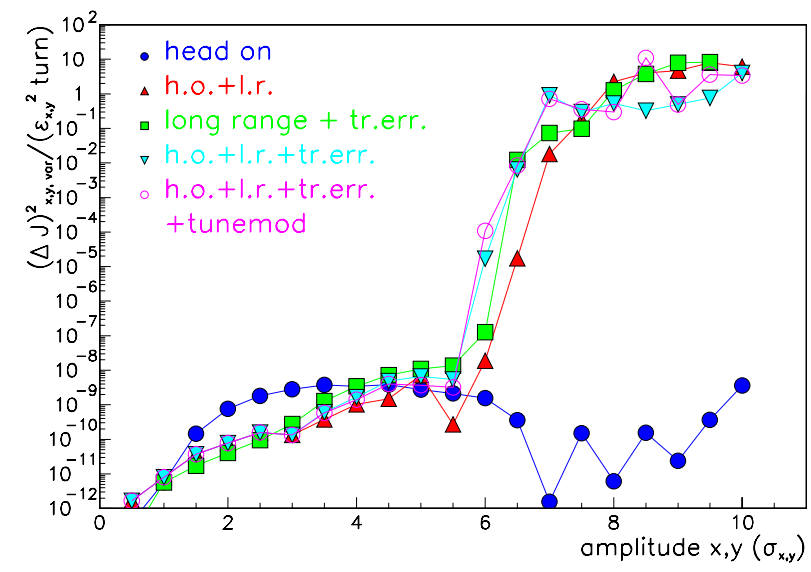

FIG. 1. (Color) The change of action variance per turn, normalized by the square transverse emittance, as a function of starting particle amplitude in the LHC, considering the motion in a 4dimensional transverse phase space (2 transverse degrees of freedom) for two interaction points with alternating horizontal/ vertical crossings and several additional sources of nonlinearity, such as long-range collisions, the uncorrected nonlinear field errors of the final-triplet quadrupoles, and a tune modulation of amplitude $10^{-4}$ [5]. The two beams, with $10^{11}$ protons per bunch, are separated by $9.5 \sigma_{x}^{\prime}$ at the long-range collision points. Whenever long-range collisions are included in the simulation, the diffusion rate increases sharply at about $6 \sigma_{x, y}$. The phase advances between the two IPs were taken to be $31.655 \times(2 \pi)$ and $29.66 \times(2 \pi)$, respectively.

To this end, we apply the Chirikov overlap criterion to a simplified model describing the long-range interactions encountered at one IP of a circular machine. Finally, we compute the locations of the most important resonances as well as a diffusion coefficient based on a Fokker-Planck type of approximation for the behavior of the chaotic trajectories. Throughout this article, we assume LHC-like parameters.

As a simplification, both in the simulation and in the analytical treatment we consider particle motion in one transverse plane only ( 1 transverse degree of freedom). This is a necessary prerequisite for applying the Chirikov criterion. All calculations, results, and figures presented in the remainder of this article refer to a 2-dimensional transverse phase space.

\section{SIMULATIONS}

We consider a single collision point, round Gaussian beams, and particle motion in the crossing plane only. The particle coordinate and slope at the IP can be written as $x=\sqrt{2 J \beta^{*}} \sin \phi$ and $x^{\prime}=\sqrt{2 J / \beta^{*}} \cos \phi$, where $(J, \phi)$ are action-angle variables, $\beta^{*}$ is the beta function at the IP (horizontal and vertical beta functions are assumed to be equal), and the prime denotes the derivative with respect to the accelerator path $s$. The long-range collisions occur at a betatron phase advance close to $\pi / 2$ from the IP. The collisions before and after the IP add up and, thus, the net effect of all long-range collisions around one IP can be represented as a single shift in the IP coordinate $x$ (e.g., see $[4,5])$. A full turn around the storage ring is described by

$$
\left(\begin{array}{c}
x \\
x^{\prime}
\end{array}\right)_{1}=\left(\begin{array}{cc}
\cos \mu & \beta^{*} \sin \mu \\
-\sin \mu / \beta^{*} & \cos \mu
\end{array}\right)\left(\begin{array}{c}
x+f\left(x^{\prime}\right) \\
x^{\prime}
\end{array}\right)_{0},
$$

where $\mu$ is the phase advance per turn,

$$
\begin{gathered}
f\left(x^{\prime}\right)=-K\left[\frac{1}{x^{\prime}+\theta_{c}}\left(1-e^{-\left[\left(x^{\prime}+\theta_{c}\right)^{2} / 2 \sigma_{x}^{\prime 2}\right]}\right)\right. \\
\left.-\frac{1}{\theta_{c}}\left(1-e^{-\theta_{c}^{2} / 2 \sigma_{x}^{\prime 2}}\right)\right],
\end{gathered}
$$

with $\theta_{c}$ the full crossing angle, $K=\left(2 r_{p} N_{b} n_{\mathrm{par}} / \gamma\right), N_{b}$ the bunch population, $n_{\mathrm{par}}$ the total number of long-range collision points on both sides of one IP, $\sigma_{x}^{\prime}$ the rms beam divergence at the main IP, and $\gamma$ the relativistic factor. Equation (2) describes the effect of a nonlinear deflection ("kick") at a long-range collision point, as viewed at a location downstream or upstream which is separated from the location of the kick by $90^{\circ}$ in betatron phase advance. Equation (1) represents the concatenation of a kick and a rotation and resembles a generalized drift. It is not strictly speaking a kick itself. Yet, we will loosely refer to it as such, since it is the image of a long-range beam-beam kick after a rotation in phase space.

Note also that instead of using the coordinates at the main (head-on) collision point, we could have chosen directly those at the long-range collision point. In that case, the new horizontal coordinate $\tilde{x}$ would have been unchanged by the opposite beam, and the new slope $\tilde{x}^{\prime}$ transformed into $\tilde{x}^{\prime}+\tilde{f}(\tilde{x})$, where $\tilde{f}(\tilde{x})$ would have been a proper kick. However, we prefer to retain Eqs. (1) and (2), in order to be consistent with an earlier publication [5], where head-on collisions were also included in the simulation, and where the present coordinate system was chosen for computational efficiency and convenience.

In Eq. (2), a static dipole kick was subtracted (the term proportional to $\left.1 / \theta_{c}\right)$, as the resulting closed-orbit distortion can be taken into account and eliminated from the analysis, simply by redefining the reference axes of the local phase-space coordinates. Finally, let us mention that the positive sign of the coefficient $K$ applies to equal-charge beams.

We have performed simulations of this model system for parameters roughly representing proton-proton collisions in the LHC, which are listed in Table I. Simulated phase-space diagrams for various bunch charges are displayed in Fig. 2, along with the corresponding diagrams of tune versus amplitude (Fig. 3), computed through Laskar's frequency map analysis method [7]. The diagrams provide a view of the resonant structure of the system in frequency space. The form of the tune curves reveals the strong nonlinearity encountered in the vicinity of the opposing beam.

The motion in phase space is regular for low bunch populations. As for an increasing bunch population the perturbation grows, a multitude of high-order resonances 

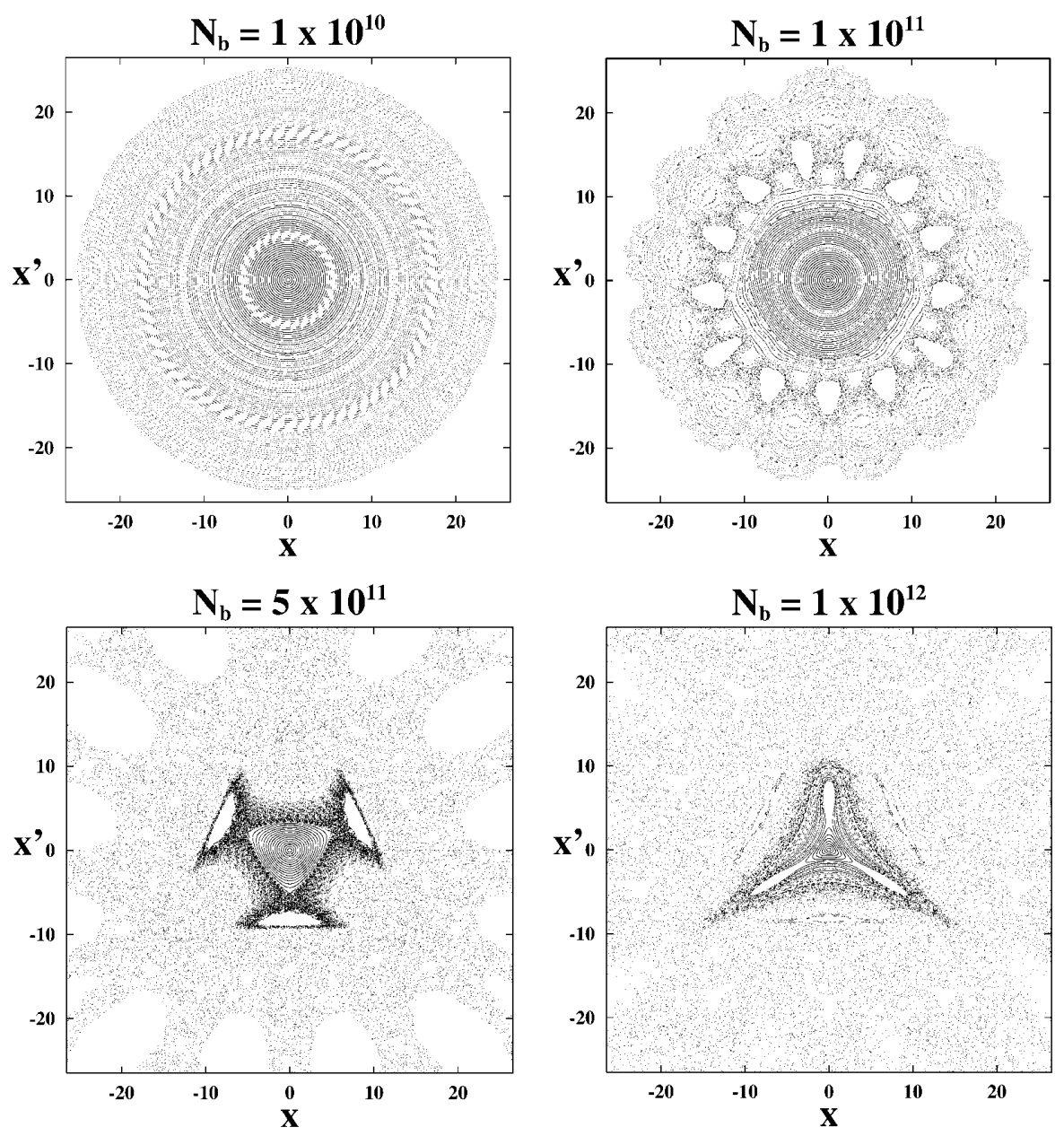

FIG. 2. Phase-space plots for various bunch populations simulated using the 2-dimensional model of the long-range beam-beam force of Eqs. (1) and (2). The transverse variables $\left(x, x^{\prime}\right)$ are measured in units of the rms beam size and rms divergence $\left(\sigma_{x}, \sigma_{x}^{\prime}\right)$.

appears (see [8], for a study on the resonance bifurcations of this class of maps). By the time $N_{b}$ reaches $5 \times 10^{11}$, most of the resonances are overlapping, giving rise to a large chaotic area, and the tune is shifted towards the thirdorder resonance. Interestingly, when the bunch population is doubled $\left(N_{b}=10^{12}\right)$, the topology of the phase space changes drastically, as the central tune passes through the third-order resonance.

If the oscillation amplitudes (expressed as slopes at the IP) are small compared with the crossing angle, and assuming that the latter is several times larger than the rms beam divergence, we can drop the exponential term in (2), and the force decreases inversely with the distance to the other beam, $r^{\prime}=\left(x^{\prime}+\theta_{c}\right)$. Because of our choice of coordinates, transverse distances at the long-range collision points are described as angles at the IP. Note that dropping the exponential term in Eq. (2) is equivalent to replacing a beam with a Gaussian distribution by a pencil beam.

Phase-space plots obtained for this simplified model are shown in Fig. 4. Although many details of the phase space are different, especially at large amplitudes, in most cases the chaotic boundary is about the same as in the simula- tions employing the exact force (Fig. 2). Figure 5 displays the corresponding curves of tune shift with amplitude.

The difference in the phase-space topology for large amplitudes arises due to a singularity in the approximate $1 / r^{\prime}$ force which is encountered when a particle passes through the center of the opposing beam, at $x^{\prime}=\theta_{c}$. In the approximate force, particles crossing that point are escaping towards infinitely large amplitudes. On the other hand, no such singularity exists in the case of the full kick, Eq. (2), due to the presence of the exponential term. In the simulation, groups of particles are launched with random nonzero initial betatron phases. Thus, the simulated particles will come close to the singularity, but usually not pass through it exactly, even if their starting amplitudes are equal to the beam separation. This singularity is of no direct concern to us, since we are interested in modeling the particle motion near the diffusive aperture, which, for the nominal LHC parameters, is significantly smaller than the beam separation. However, we keep in mind that at much lower bunch currents our approximation is no longer self-consistent, when the diffusive aperture approaches the singularity. 

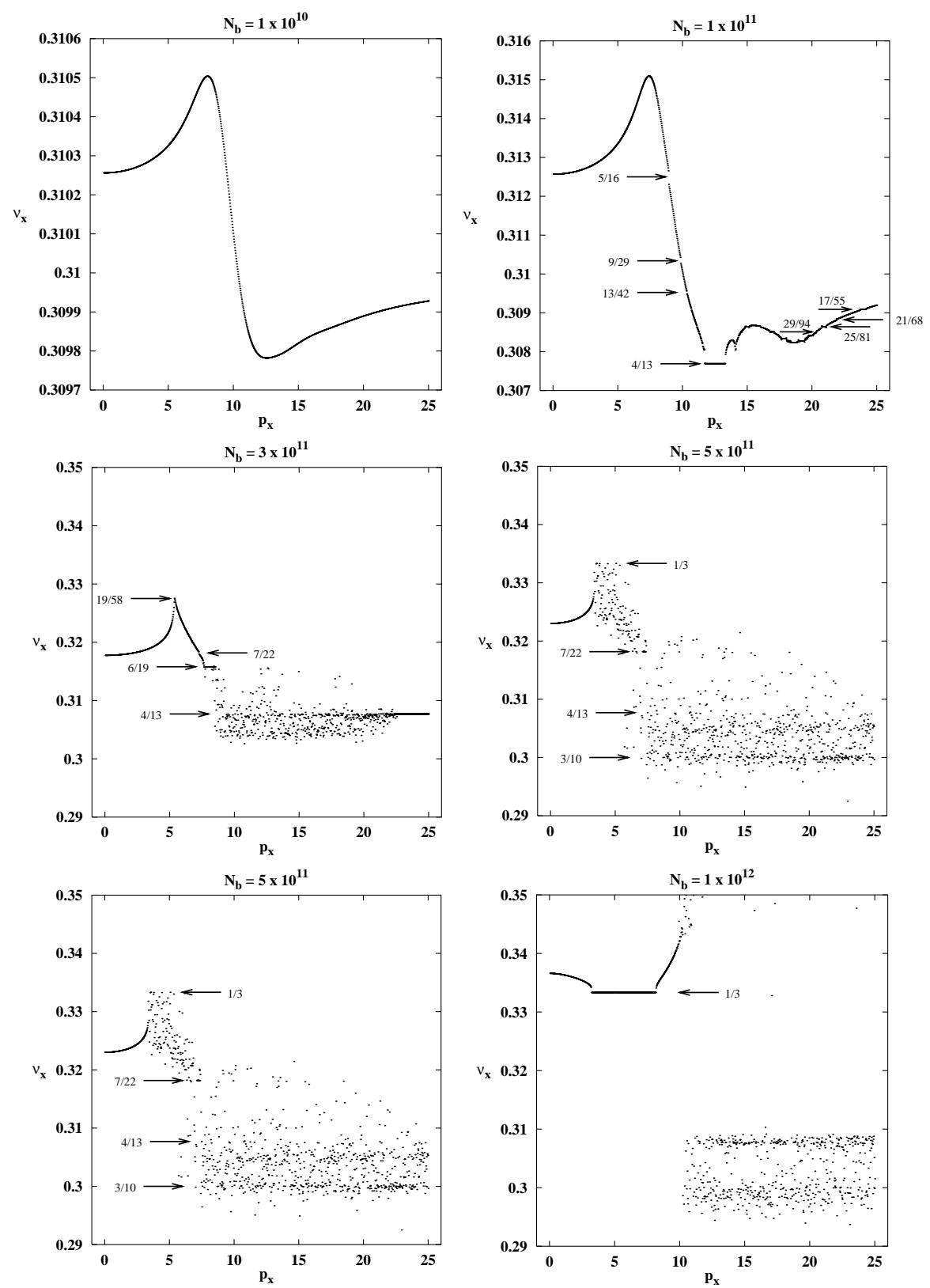

FIG. 3. Tune shifts versus initial particle slope, measured in units of the LHC rms beam divergence, for different bunch populations. The tracking was done using the 2-dimensional model of the long-range beam-beam force, Eq. (1).

Figure 6 compares diffusion rates, i.e., the increase in the action variance of a group of particles per unit time, simulated using the exact and the approximated long-range beam-beam force as a function of the bunch population.

As we have done previously for the 4-dimensional simulation results of Fig. 1, we identify the steep increases that are visible in Fig. 6 with the diffusive aperture. The values of the diffusive aperture thus obtained are summarized in Fig. 7, for both the exact force and for the $1 / r^{\prime}$ approximation. The figure illustrates that for bunch intensities above $5 \times 10^{11}$ the $1 / r^{\prime}$ approximation works well. For lower intensities, this approximation gives a smaller diffusive aperture than the exact simulation, and, thus, it can be used as a "worst case" estimate.

Even for high intensities the simulated diffusion rates $\left\langle(\Delta J)^{2}\right\rangle$ per turn at amplitudes larger than the diffusive aperture are not precisely the same in the two pictures of Fig. 6. This difference is understandable and indeed expected as the nature of diffusion for the two problems is quite different: the chaotic behavior for the $1 / r^{\prime}$ force is associated with particles escaping to infinity, whereas the chaotic phase-space region for the full problem is bounded.

Note that in Fig. 7 the diffusive aperture first decreases as a function of bunch population $N_{b}$, and then it rises again for $N_{b}$ larger than about $6 \times 10^{11}$. This is consistent 

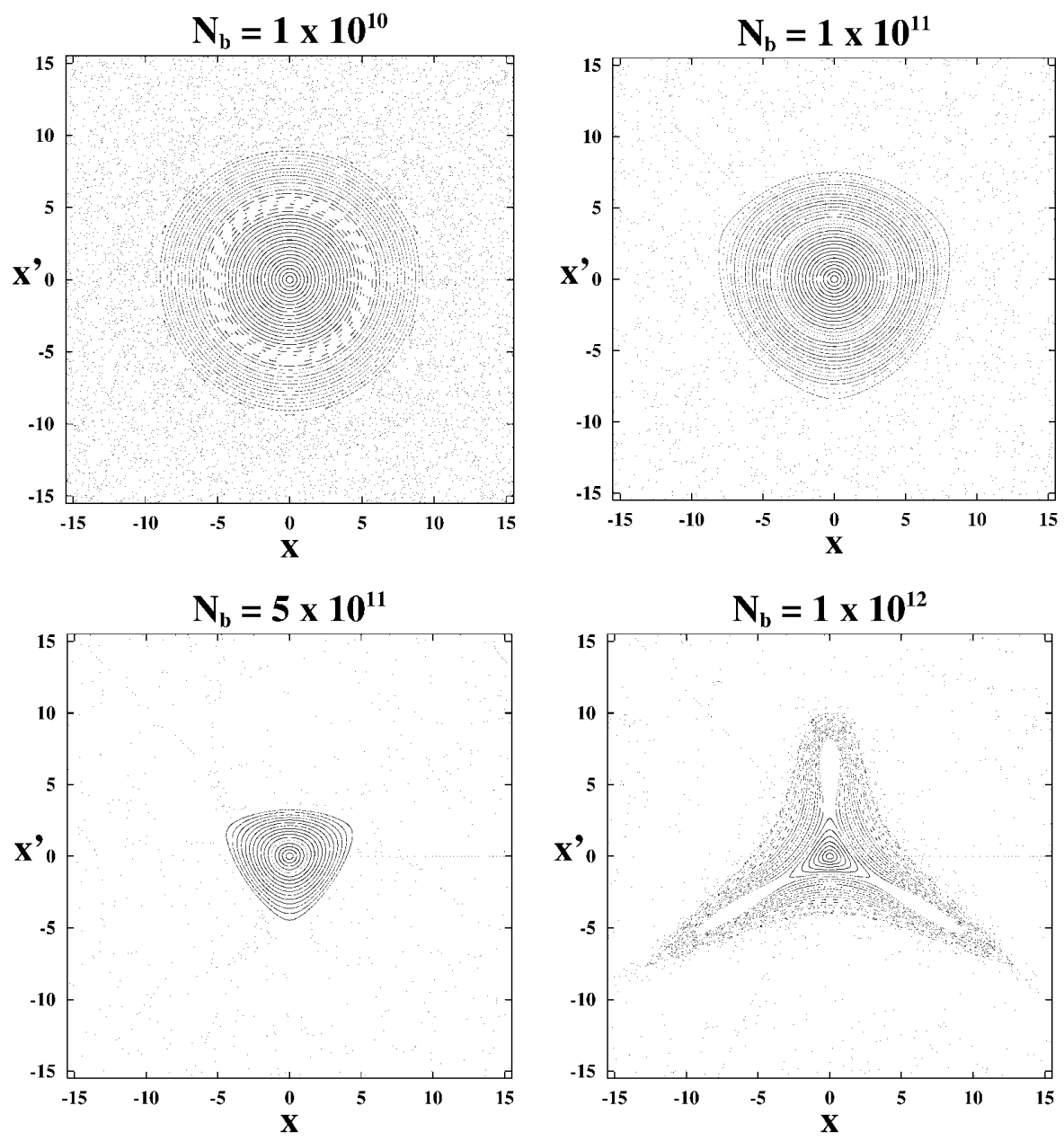

FIG. 4. Phase space for various bunch populations simulated using a simplified model of motion in one transverse plane for the long-range beam-beam force, Eq. (1) and the $1 / r^{\prime}$ approximation to Eq. (2). The transverse variables are measured in units of the rms beam size $\sigma_{x}$ and beam divergence $\sigma_{x}^{\prime}$.

with the phase-space diagrams in Figs. 2 and 4, as well as with the simulated tune shifts versus amplitude in Figs. 3 and 5. In our example, a decisive ingredient determining the diffusive aperture is the third-integer resonance, whose location moves inwards for increasing bunch population. Above a certain current, the third-order resonance islands become stable and so does the particle motion in a fairly large region of phase space. This phenomenon indeed depends on the working point.

\section{HAMILTONIAN, DETUNING, AND DRIVING TERMS}

The Hamiltonian corresponding to Eq. (1) consists of a periodic series of long-range kicks and linear rotations

$$
H(J, \phi, \theta)=Q_{0} J+V(J, \phi) \frac{1}{2 \pi} \sum_{p} e^{i p \theta},
$$

where $Q_{0}$ is the unperturbed tune and the series representing the Fourier expansion of the Dirac $\delta$ function, expressing the localization of the beam-beam kick in a single IP encountered on successive turns. The beam-beam poten- tial $V(J, \phi)=V\left[x^{\prime}(J, \phi)\right]$ can be calculated by integrating the beam-beam kick $f\left(x^{\prime}\right)$ from $-\infty$ to $x^{\prime}$. We obtain

$$
\begin{gathered}
V\left(x^{\prime}\right)=\frac{K}{2}\left[E_{i}\left(-\frac{\left(x^{\prime}+\theta_{c}\right)^{2}}{2 \sigma_{x^{\prime}}^{2}}\right)-\ln \left(\frac{\left(x^{\prime}+\theta_{c}\right)^{2}}{2 \sigma_{x^{\prime}}^{2}}\right)\right. \\
\left.+\frac{2 x^{\prime}}{\theta_{c}}\left(1-e^{-\theta_{c}^{2} / 2 \sigma_{x^{\prime}}^{2}}\right)\right]
\end{gathered}
$$

where $E_{i}$ is the exponential integral,

$$
E_{i}(u)=\int_{-\infty}^{u} \frac{e^{u^{\prime}}}{u^{\prime}} d u^{\prime}
$$

representing the exponential part of the force. The change in the position of a particle at the main IP, due to the generalized drift representing the combined action of upstream and downstream long-range kicks, is related to the partial derivative of $V$ with respect to $x^{\prime}$, i.e., $\Delta x(\theta)=$ $\partial V / \partial x^{\prime} \sum_{n} \delta(\theta-n 2 \pi)$, and $x^{\prime}$ can again be expressed in terms of action-angle variables via $x^{\prime}=\sqrt{2 J / \beta^{*}} \cos \phi$.

Near a resonance $n Q \approx p$ of order $n$, we may approximate the Hamiltonian (see, e.g., [9]) as

$$
H_{r} \approx Q_{0} J+g(J)+h_{n} \cos (n \phi-p \theta) .
$$



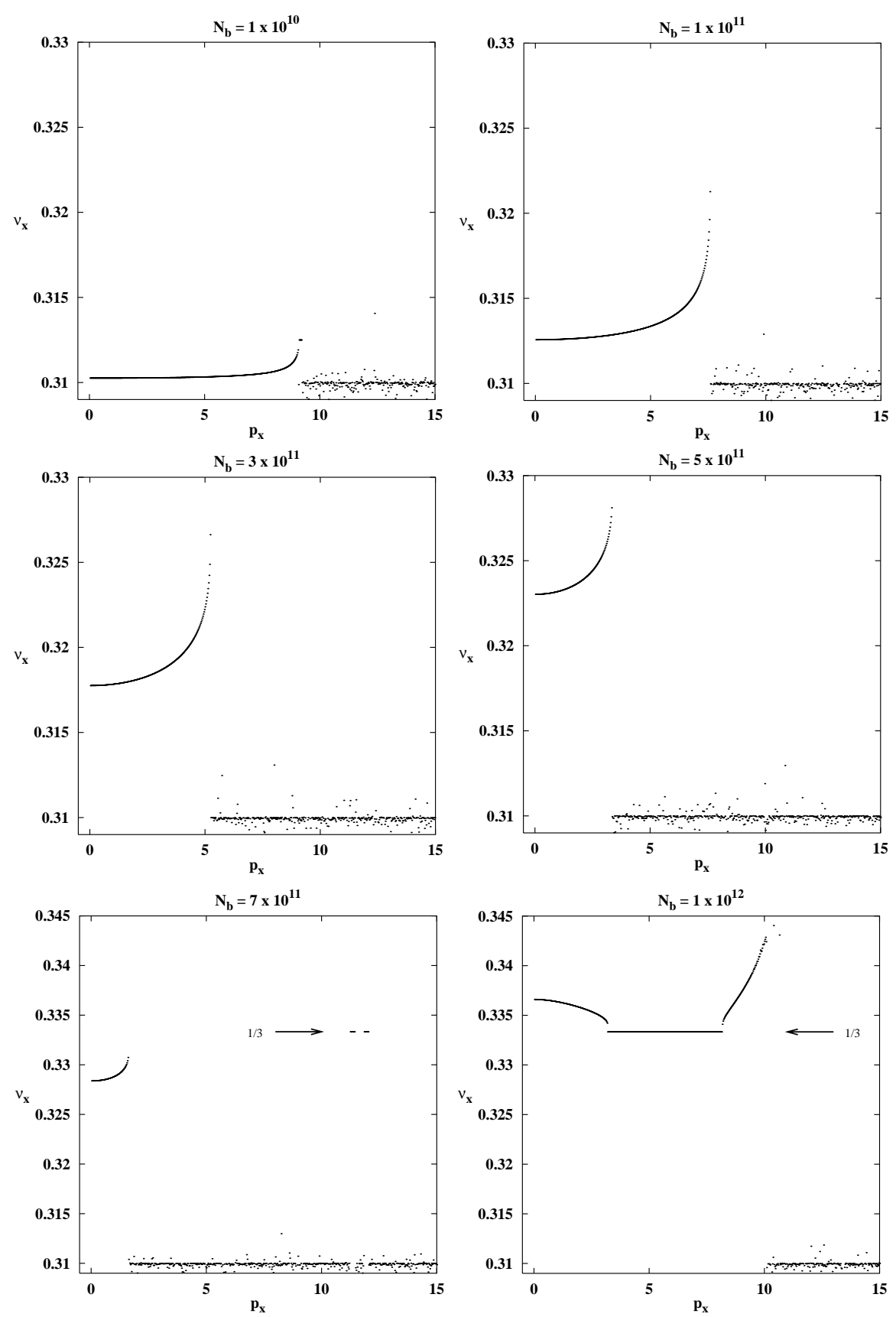

FIG. 5. Tune shifts versus initial particle slope, measured in units of the LHC rms beam divergence, for different bunch populations. The tracking has been done using the simplified of motion in one transverse plane for the long-range beam-beam force, Eq. (1) and the $1 / r^{\prime}$ approximation to Eq. (2).

The shift of the tune with amplitude in first-order perturbation theory is

$$
\frac{d g(J)}{d J}=\frac{1}{2 \pi}\left\langle\frac{\partial V}{\partial J}\right\rangle_{\phi},
$$

where the angular brackets $\langle\cdots\rangle_{\phi}$ denote an average over the angle variable. The driving term $h_{n}$ is given by the Fourier transform of the potential, i.e.,

$$
h_{n}(J)=\frac{1}{2 \pi^{2}} \int_{0}^{2 \pi} V(J, \phi) \cos (n \phi) d \phi .
$$

The usual procedure followed for integrals involving exponentials with arguments of trigonometric functions is to expand them into a series of Bessel functions (see, e.g., [10]):

$$
\begin{aligned}
e^{z \cos \theta} & =I_{0}(z)+2 \sum_{n=1}^{+\infty} I_{n}(z) \cos (n \theta) \\
& =\sum_{n=-\infty}^{+\infty} I_{n}(z) \cos (n \theta)
\end{aligned}
$$


where the symbols $I_{n}$ represent the $n$th order modified Bessel functions of the first kind. Calculation of the integrals yields

$$
\begin{aligned}
\frac{d g(J)}{d J}=\frac{K}{2 \pi \beta^{*} \sqrt{\theta_{c}^{2}-2 J / \beta^{*}}} & {\left[\frac{1}{\theta_{c}+\sqrt{\theta_{c}^{2}-2 J / \beta^{*}}}+\frac{e^{-\left(\theta_{c}^{2} / 2 \sigma_{x^{\prime}}^{2}\right)-\left(J / 2 \beta^{*} \sigma_{x^{\prime}}^{2}\right)}}{4 \sqrt{2 J / \beta^{*}}}\right.} \\
& \left.\times \sum_{k, l=-\infty}^{\infty} I_{k}\left(-\frac{J}{2 \beta^{*} \sigma_{x^{\prime}}^{2}}\right) I_{l}\left(-\frac{\theta_{c}}{\sigma_{x^{\prime}}^{2}} \sqrt{\frac{2 J}{\beta^{*}}}\right) \mathcal{D}_{k, l}(J)\right],
\end{aligned}
$$

where

$$
\begin{aligned}
\mathcal{D}_{k, l}(J)= & \mathcal{R}^{|2 k+l+1|}+\mathcal{R}^{|2 k+l-1|} \\
& +\mathcal{R}^{|2 k-l+1|}+\mathcal{R}^{|2 k-l-1|},
\end{aligned}
$$

and

$$
\mathcal{R}(J)=-\frac{\sqrt{2 J / \beta^{*}}}{\sqrt{\theta_{c}^{2}-2 J / \beta^{*}}+\theta_{c}} .
$$

The convergence behavior of the expansion in Bessel functions is illustrated in Fig. 8, where we plot the
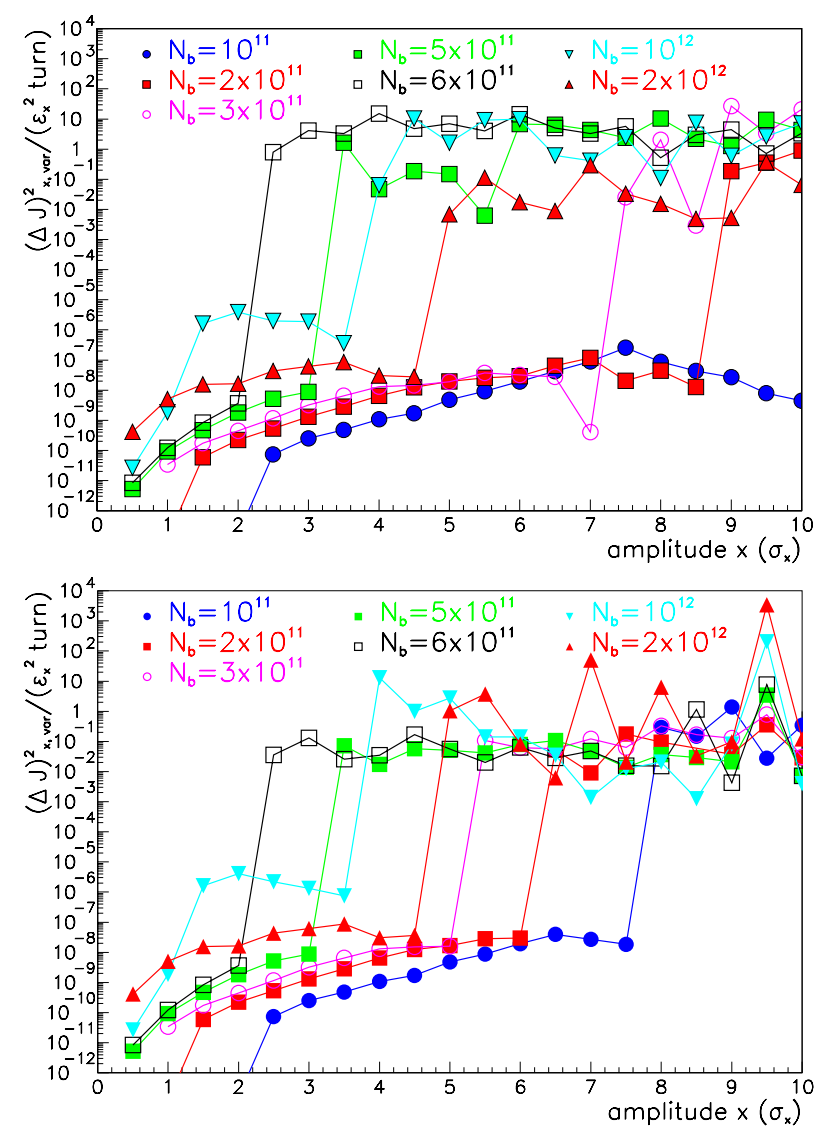

FIG. 6. (Color) The change of action variance per turn, in units of the LHC rms design emittance $0.5 \mathrm{~nm}$, as a function of starting amplitude for a simulation in one transverse plane (i.e., $y^{\prime}=0$ ) with $9.5 \sigma_{x}^{\prime}$ separation, considering a single interaction point with the beam-beam crossing in the plane of motion. The top picture shows results for the exact beam-beam force; the bottom picture shows those for the $1 / r^{\prime}$ approximation. The various curves correspond to different bunch populations. dependence of the analytical detuning estimate (5) at an amplitude close to $9 \sigma_{x}^{\prime}$ as a function of the maximum values $k$ and $l$, for which the corresponding Bessel functions are still taken into account. The partial sums for $|k|_{\max }$ and $|l|_{\max }$ about equal to 30 and 50, respectively, are constant up to machine precision. However, the violent oscillations of these partial sums at lower order indicate that they are numerically ill conditioned, which may degrade the accuracy of the result despite the convergence.

The first term in the square brackets of (5) corresponds to the $1 / r^{\prime}$ approximation. This part of the detuning can be derived using formula (3.613.1) of [11]. The term in front of the square brackets diverges at an amplitude which is $2-3 \sigma_{x}^{\prime}$ smaller than the separation of the two beams (expressed as the crossing angle) and so does the $1 / r^{\prime}$ approximation, as illustrated in Fig. 9. This divergence indeed indicates the breakdown of the validity of the $1 / r^{\prime}$ approximation. It is not immediately evident that the term in square brackets cancels the divergence in front, nor that it necessarily should, since we are applying a first-order perturbative treatment.

Regardless, the full expression more closely approaches the simulated behavior of tune versus amplitude, though above a certain amplitude it equally fails to reproduce the simulation result. We attribute the remaining discrepancy to either the first-order nature of the analytical estimate

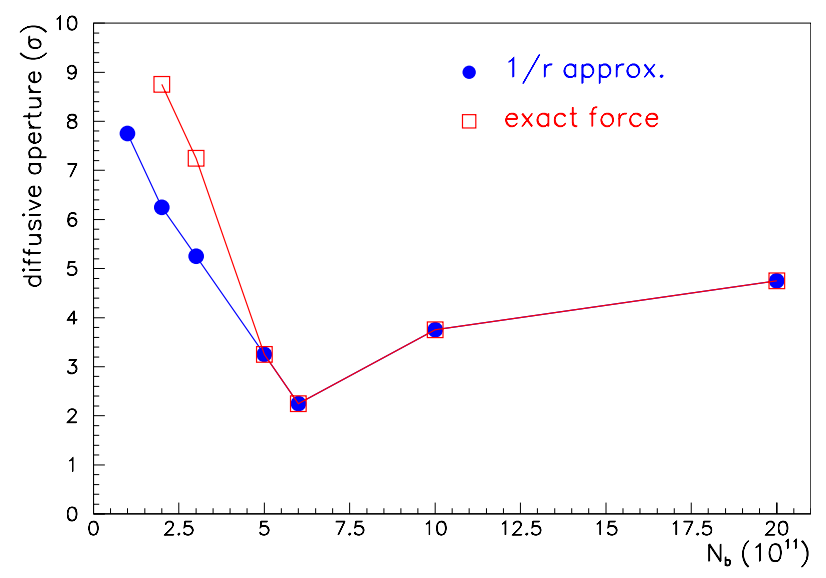

FIG. 7. (Color) Simulated diffusive aperture as a function of bunch population for 30 parasitic collision points and $\theta_{c} \approx$ $9.5 \sigma_{x^{\prime}}$. The figure compares the results for the exact force and those obtained using the $1 / r^{\prime}$ approximation. Only particle motion in the plane of crossing is simulated ( 1 transverse degree of freedom). 

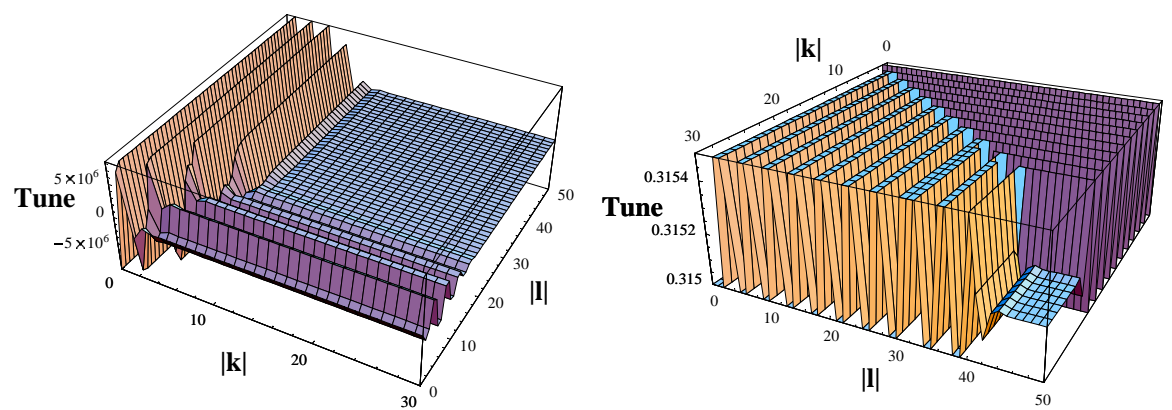

FIG. 8. (Color) Tune shift calculated using Eq. (5) at a large amplitude (near $9 \sigma$, close to the crossing with opposing beam) versus the order of the terms included in the Bessel function series expansion. The plot on the right is a zoom of the left one from a different perspective.

or to the limited computing precision when evaluating the double sum over the product of Bessel functions.

Using again the expansion (4), or employing the formula (4.397.6) of [11] $\left[\int_{0}^{\pi} \ln \left(1-2 a \cos x+a^{2}\right) \cos n x d x=\right.$ $-\pi /\left(n a^{n}\right)$ for $\left.a^{2}>1\right]$, and considering $n>1$, the resonance driving term can be written as

$$
\begin{aligned}
h_{n}(J)=\frac{2 K}{\pi n}[ & (-\mathcal{R})^{n}+\frac{\sqrt{2 J / \beta^{*}} e^{-\left(\theta_{c}^{2} / 2 \sigma_{x}^{\prime 2}\right)-\left(J / 2 \beta^{*} \sigma_{x}^{\prime 2}\right)}}{8 \theta_{c} \sqrt{\theta_{c}-2 J / \beta^{\star}}} \\
& \times \sum_{k, l=-\infty}^{\infty} I_{k}\left(-\frac{J}{2 \beta^{*} \sigma_{x}^{\prime 2}}\right) \\
& \left.\times I_{l}\left(-\frac{\theta_{c}}{\sigma_{x}^{\prime 2}} \sqrt{\frac{2 J}{\beta^{*}}}\right) \mathcal{D}_{k, l, n}^{\prime}\right]
\end{aligned}
$$

where

$$
\begin{aligned}
\mathcal{D}_{k, l, n}^{\prime}(J)= & -\mathcal{R}^{|2 k+l+n+1|}+\mathcal{R}^{|2 k+l-n+1|} \\
& -\mathcal{R}^{|2 k+l-n-1|}+\mathcal{R}^{|2 k-l-n+1|}-\mathcal{R}^{|2 k-l+n+1|} \\
& +\mathcal{R}^{|2 k+l+n-1|}-\mathcal{R}^{|2 k-l-n-1|}+\mathcal{R}^{|2 k-l+n-1|}
\end{aligned}
$$

Again, the first term in the square brackets represents the $1 / r^{\prime}$ force.

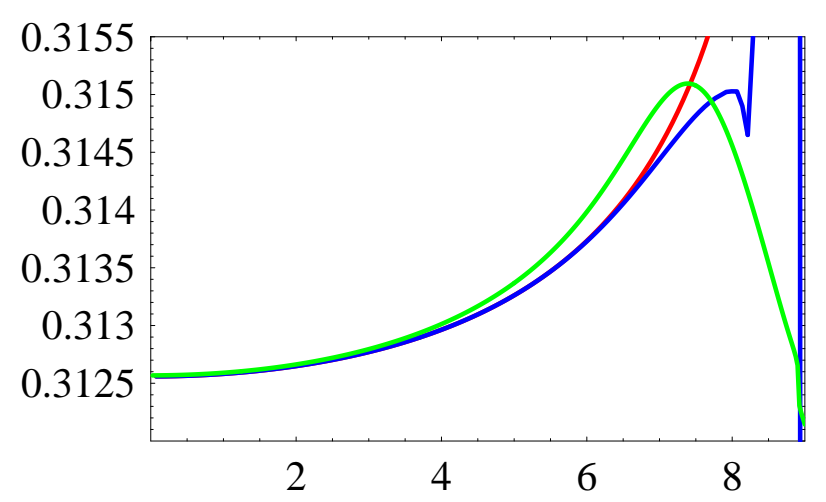

FIG. 9. (Color) Tune as a function of amplitude in units of $\sigma_{x}^{\prime}$ due to long-range beam-beam interaction for a separation of $9.5 \sigma_{x}^{\prime}$. The tracking result (green), the $1 / r^{\prime}$ term only (red), and the full force, where the Bessel function series of (5) are expanded to maximum absolute orders $|k|_{\max }=30$ and $|l|_{\max }=$ 50 (blue).

\section{OVERLAP CRITERION}

For simplicity, we now restrict the analysis entirely to the $1 / r^{\prime}$ approximation. The resonance half-width of the $n$th order resonance is $\Delta J_{n, 1 / 2}=2\left(\frac{h_{n}}{d^{2} g / d J^{2}}\right)^{1 / 2}$ and the distance between two resonances of order $n_{1}$ and $n_{2}\left(n_{2}>\right.$ $\left.n_{1}\right)$ is $\delta J=\left[\frac{1}{n_{1}}-\frac{1}{n_{2}}\right] \frac{1}{\left|d^{2} g / d J^{2}\right|}$. Strong chaos occurs if two adjacent resonances overlap [12]:

$$
\frac{2}{3} \delta J \leq \Delta J_{n_{1}, 1 / 2}+\Delta J_{n_{2}, 1 / 2} .
$$

The factor $2 / 3$ accounts for the width of the separatrix and for higher-order islands $[9,12]$. This condition can be rewritten as

$$
\frac{1}{3}\left(\frac{1}{n_{1}}-\frac{1}{n_{2}}\right) \leq\left(\sqrt{h_{n_{1}}}+\sqrt{h_{n_{2}}}\right) \sqrt{\left|\frac{d^{2} g}{d J^{2}}\right|} .
$$

As a first example, we consider $n_{2}=\left(n_{1}+1\right)$, assume $h_{n_{1}} \approx h_{n_{2}}$, and insert the expressions for $d g / d J$ and $h_{n}$ derived in Eqs. (5) and (6). The nonlinear equation describing the threshold of instability then becomes

$$
\begin{gathered}
\left(\frac{1+2 \sqrt{1-A^{2}}}{\left(1-A^{2}\right)^{3 / 2}\left(1+\sqrt{1-A^{2}}\right)^{2}}\right)\left(\frac{A}{1+\sqrt{1-A^{2}}}\right)^{n_{\mathrm{res}}} \\
\geq \frac{\pi^{2} \theta_{c}^{4} \beta^{* 2}}{36 K^{2}} \frac{1}{n_{\mathrm{res}}\left(n_{\mathrm{res}}+1\right)^{2}},
\end{gathered}
$$

where $A \equiv \sqrt{2 J / \beta^{*}} / \theta_{c}$ is the particle amplitude normalized to the separation, and $n_{\text {res }}=n_{1}$. The overlap criterion is necessary, but not sufficient. In order to observe chaos, resonances of order $n_{\text {res }}$ also need to be present near the threshold amplitude $A$. This depends on the nominal tune and on the detuning $d g / d J$. As a worst case, we may assume that a resonance of a given order $n_{\text {res }}$ is located directly at the boundary expressed by Eq. (7), where overlap would occur.

An important point to note is that the action variable $J_{\mathrm{ov}, n_{\mathrm{res}}} \equiv A \beta^{*} \theta_{c}^{2} / 2$ for which the overlap criterion (7) is fulfilled should be taken as the action value at the center of the island, the destruction of whose separatrix gives rise to chaos and diffusion, whereas the actual diffusive aperture corresponds to an action variable obtained from $J_{\mathrm{ov}, n_{\mathrm{res}}}$ by subtracting the resonance half-width $\Delta J_{n_{\text {res }, 1 / 2}}$ : 


$$
J_{\mathrm{da}} \approx J_{\mathrm{ov}, \mathrm{n}_{\mathrm{res}}}-\Delta J_{n_{\mathrm{res}}, 1 / 2} .
$$

Numerical solutions of (7) and (8) are shown in Fig. 10 illustrating the dependence of the overlap amplitude $x_{\mathrm{da}}=$ $\sqrt{2 J_{\mathrm{da}} / \varepsilon}$ (where $\varepsilon$ is the geometric rms emittance) on the resonance order, the bunch population, and the crossing angle.
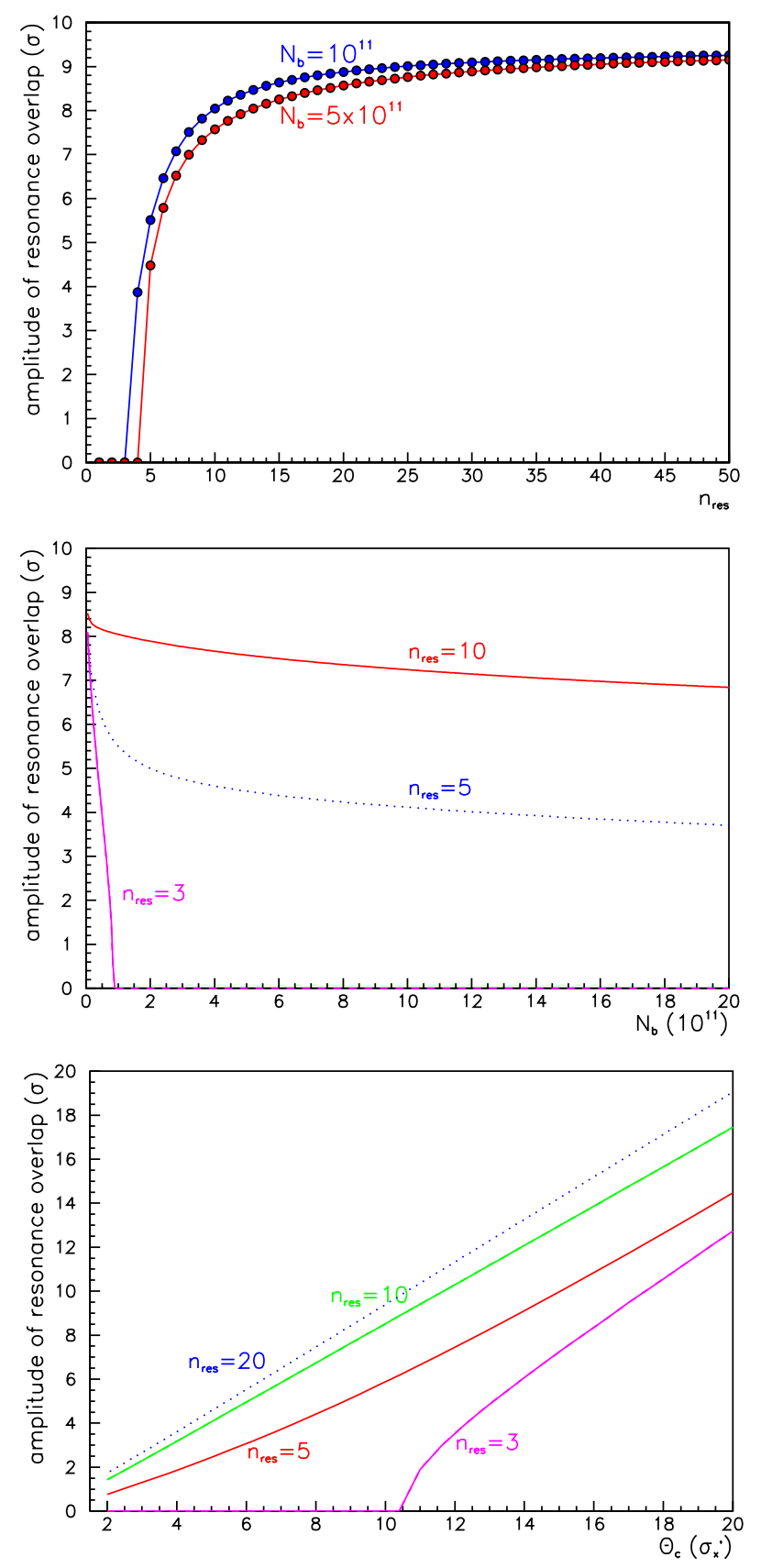

FIG. 10. (Color) Minimum amplitude at which the overlap condition Eq. (7) is fulfilled as a function of (top) resonance order $n_{\mathrm{res}}$ (for $\theta_{c} \approx 9.5 \sigma_{x}^{\prime}, N_{b}=1.05 \times 10^{11}$ ), (center) bunch population (for the same crossing angle and three different resonance orders), and (bottom) crossing angle $\theta_{c}$ (for a bunch population of $N_{b}=1.05 \times 10^{11}$ and five different resonance orders).
On closer inspection, the numerical simulations indicate that chaos occurs due to the overlap of a resonance of low order, such as third order, with a second considerably higher resonance.

To obtain a better analytical estimate, as a second example, we therefore consider $n_{1} \equiv n_{\text {res }}$ to be a low-order resonance, e.g., $n_{1}=3$, and $n_{2}$ to be a resonance of much
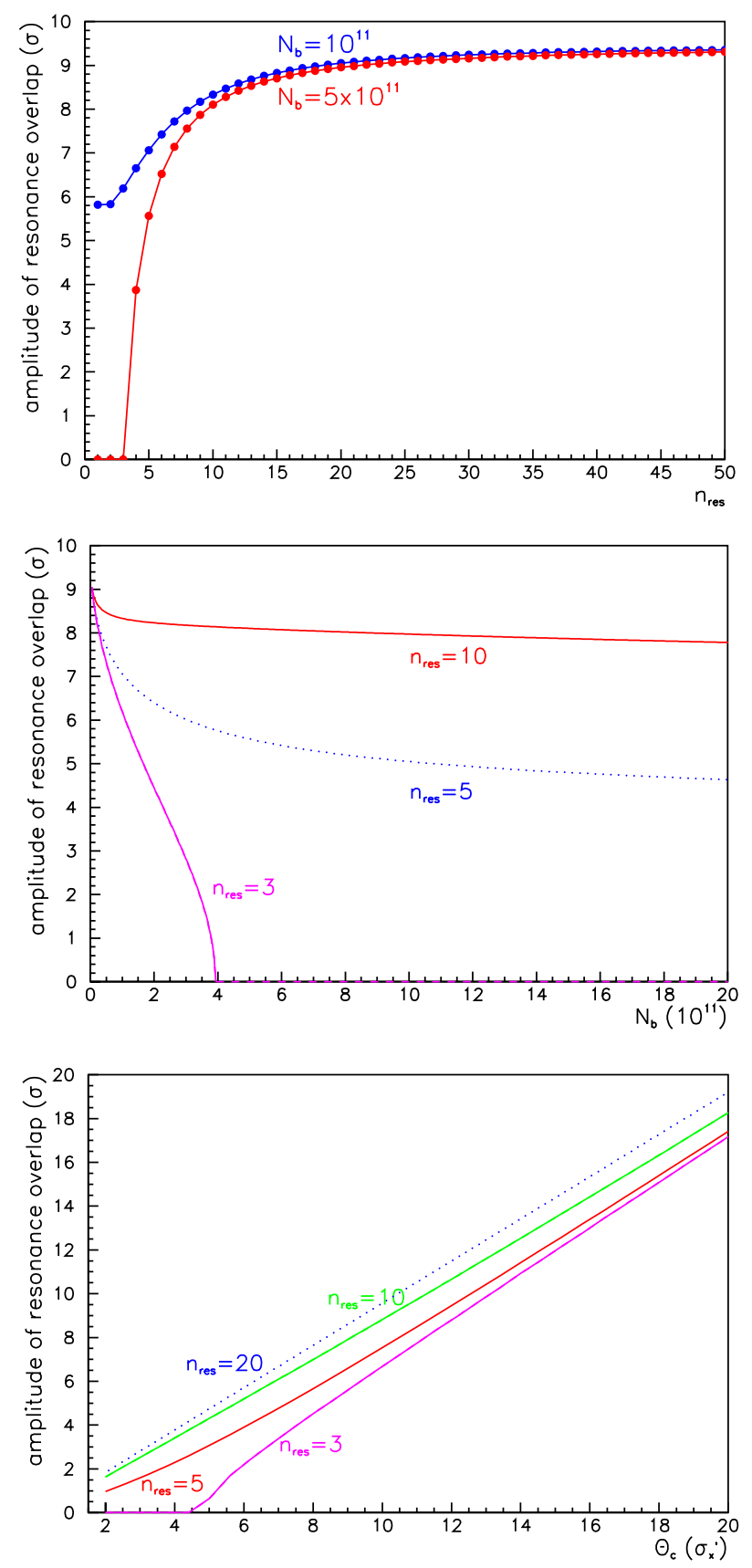

FIG. 11. (Color) Minimum amplitude at which the modified overlap condition Eq. (9) is fulfilled as a function of (top) resonance order $n_{\text {res }}$ (for $\theta_{c} \approx 9.5 \sigma_{x}^{\prime}, N_{b}=1.05 \times 10^{11}$ ), (center) bunch population (for the same crossing angle and three different resonance orders), and (bottom) crossing angle $\theta_{c}$ (for the nominal bunch population $N_{b}=1.05 \times 10^{11}$ and five different resonance orders). 
higher order. We can then neglect its contribution in the difference $1 / n_{1}-1 / n_{2}$ and in the sum of the two resonance widths, and we obtain, as a revised expression for the chaotic threshold, the inequality

$$
\begin{gathered}
\left(\frac{1+2 \sqrt{1-A^{2}}}{\left(1-A^{2}\right)^{3 / 2}\left(1+\sqrt{1-A^{2}}\right)^{2}}\right)\left(\frac{A}{1+\sqrt{1-A^{2}}}\right)^{n_{\mathrm{res}}} \\
\geq \frac{\pi^{2} \theta_{c}^{4} \beta^{* 2}}{9 K^{2}} \frac{1}{n_{\mathrm{res}}^{2}} .
\end{gathered}
$$

Results computed using Eqs. (8) and (9) are displayed in Fig. 11.

Figures 10 and 11 illustrate that for a bunch population $^{1} N_{b}=1.05 \times 10^{11}$, the diffusive aperture predicted by Eqs. (7) and (9) is rather sensitive to the resonance order. For high resonance orders or large crossing angles, and also for small bunch populations, the model predicts the onset of global chaos at amplitudes where particles pass close to the center of the opposing beam (at $9.5 \sigma_{x}^{\prime}$ ). At these amplitudes, however, the $1 / r^{\prime}$ approximation can no longer be applied, and, therefore, the real diffusive aperture might either be larger or not exist at all. However, for increasing strength of the perturbation, resonances of lower order induce global chaos at significantly smaller amplitudes, where our approximate solution is selfconsistent. For a working point at $Q_{0}=0.31$ and bunch charges above $3 \times 10^{11}$, the third-order resonance determines the position of the diffusive aperture, as is evident from Fig. 3.

It is noteworthy that for a constant normalized crossing angle $\theta_{c} / \sqrt{\varepsilon / \beta^{*}}$, the diffusive aperture in units of the rms beam size depends only on the variables $\theta_{c}, \beta^{*}$ and on the perturbation parameter $K$ in the combination

$$
\frac{\theta_{c}^{2} \beta^{*}}{K} \propto \frac{\gamma \theta_{c}^{2} \beta^{*}}{N_{b} n_{\mathrm{par}}} \propto \frac{\gamma \varepsilon}{N_{b} n_{\mathrm{par}}} .
$$

\section{RESONANCE LOCATION}

The simulated phase-space diagrams in Figs. 2 and 4 indicate that the diffusive aperture for the nominal LHC working point is dominated by resonances of fairly low order. Under this assumption, we have also obtained a good agreement with the analytical overlap criterion. We can further improve the estimate based on Eqs. (8) and (9), if we take into account the actual resonance positions.

Invoking again the $1 / r^{\prime}$ approximation, the detuning function $g(J)$ gives the location in action of a resonance $(Q=p / n)$ as

$$
Q_{0}+\frac{d g}{d J}\left(J_{n, p}\right)=\frac{p}{n}
$$

or

\footnotetext{
${ }^{1}$ Recently the nominal LHC bunch intensity was changed to $1.1 \times 10^{11}$.
}

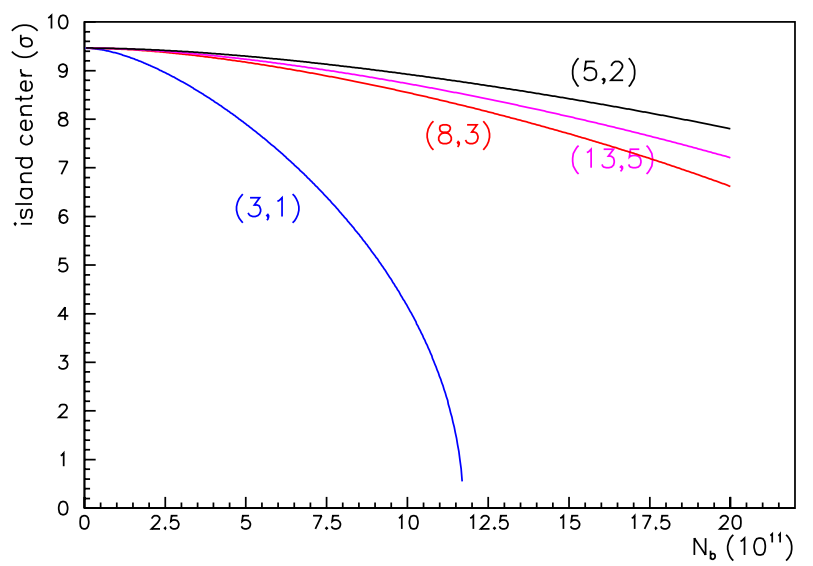

FIG. 12. (Color) Location for resonances of order 3, 5, 8, and 13 , according to Eq. (12), as a function of bunch population, for a base tune of 0.31 .

$$
\begin{aligned}
J_{n, p}= & \frac{1}{4 \pi\left(p-n Q_{0}\right)} \\
& \times\left\{-K n+\pi\left(p-n Q_{0}\right) \beta \theta_{c}^{2}+\sqrt{\pi}\right. \\
& \left.\times \sqrt{\left(p-n Q_{0}\right) \beta \theta_{c}^{2}\left[2 K n+\pi\left(p-n Q_{0}\right) \beta \theta_{c}^{2}\right]}\right\} .
\end{aligned}
$$

Using Eq. (12), we can compute the resonance locations as a function of the bunch population, as illustrated in Fig. 12 for resonances of order 3, 5, 8, and 13 .

If a resonance $n Q \approx p$ of order $n_{\text {res }}=n$ limits the diffusive aperture, we may estimate the latter by

$$
J_{\mathrm{da}} \approx J_{n, p}-\Delta J_{n, p, 1 / 2}
$$

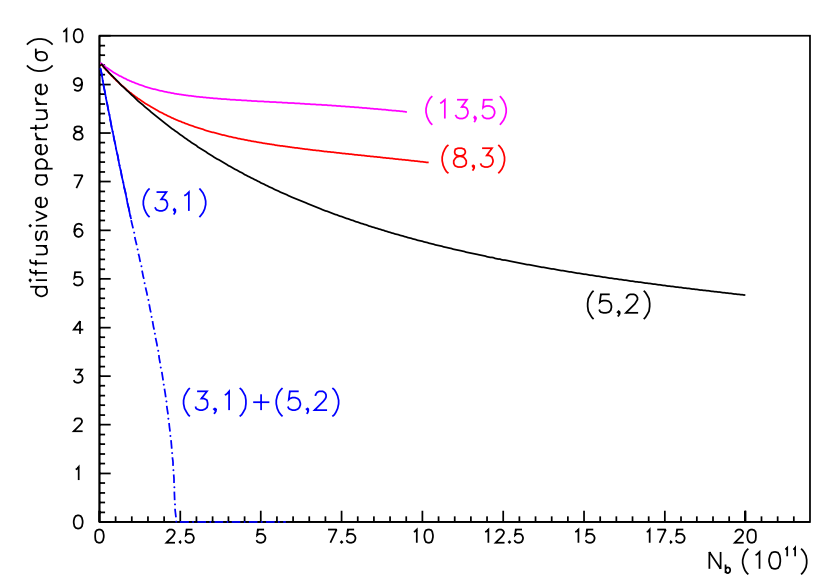

FIG. 13. (Color) Diffusive aperture corresponding to the minimum boundary for resonance islands of order $3,5,8$, and 13 , as a function of bunch population, taking into account the actual location of the resonances for a base tune of 0.31. Aperture values are shown only for bunch currents at which overlap with a high-order resonance occurs according to Eq. (9). If overlap with other low-order resonances is taken into account curves may extend farther to the right. The dotted line considers as an example the overlap between the third- and the fifth-order resonance. 
where $\Delta J_{n, p, 1 / 2}$ denotes the half-width of the $n$th order resonance evaluated for a fixed point at $J_{n, p}$. Applying Eq. (13), we obtain the diffusive aperture estimates of Fig. 13. Values are shown only for bunch populations and amplitudes at which the Chirikov criterion, Eq. (9), is fulfilled. Here, we have considered only the overlap with a high-order resonance. The dotted line illustrates that overlap with other low-order resonances may continue to significantly larger bunch populations.

We recall that at small bunch populations, for which the diffusive aperture approaches the separation, the calculation is no longer self-consistent.

\section{DIFFUSION COEFFICIENT}

The action variable is related to $x$ and $x^{\prime}$ via

$$
J=\frac{x^{2}+\left(\beta^{*} x^{\prime}\right)^{2}}{2 \beta^{*}},
$$

which follows from the relations

$$
x=\sqrt{2 J \beta^{*}} \sin \phi, x^{\prime}=\sqrt{2 J / \beta^{*}} \cos \phi .
$$

The change in action due to a long-range kick is

$$
\Delta J=\frac{2 x f\left(x^{\prime}\right)+f\left(x^{\prime}\right)^{2}}{2 \beta^{*}} .
$$

In the chaotic region of phase space, the action diffusion coefficient per turn can be estimated by averaging over the quasirandomly varying betatron phase variable as [9]

$$
\begin{aligned}
D(J)= & \frac{1}{2 \pi} \int_{0}^{2 \pi} d \phi[\Delta J(\phi)]^{2} \\
= & \frac{1}{2 \pi} K^{2} A^{4} \int_{0}^{2 \pi} d \phi\left[\frac{\sin ^{2} \phi \cos ^{2} \phi}{(1+A \cos \phi)^{2}}\right] d \phi \\
= & \frac{K^{2}}{2} \frac{1}{A-1}\left[A^{3}-A^{2}+4 A^{2} \sqrt{\frac{1-A}{1+A}}-6 A\right. \\
& \left.+6-6 \sqrt{\frac{1-A}{1+A}}\right],
\end{aligned}
$$

where $A \equiv \sqrt{2 J / \beta^{*}} / \theta_{c}$. Note that the expression in the square brackets becomes zero at $A=0$ and $A=1$, while it is real and negative for intermediate values of $A$, thus defining the validity limit of the estimate. Beyond this limit, for $A>1$, the expression becomes complex and has no physical significance.

The diffusion coefficient for various bunch populations is plotted in Fig. 14 as a function of amplitude. Comparison with Fig. 6 demonstrates that the analytical formula gives a reasonable estimate for the diffusion rate in the chaotic region, i.e., at amplitudes larger than the diffusive aperture. For smaller amplitudes, the simulated diffusion in Fig. 6 is much smaller than that computed from (17). The reason for the discrepancy at small amplitudes is that the assumption underlying Eq. (17), namely, that the motion is stochastic and the betatron phase random over long time scales, no longer holds true.

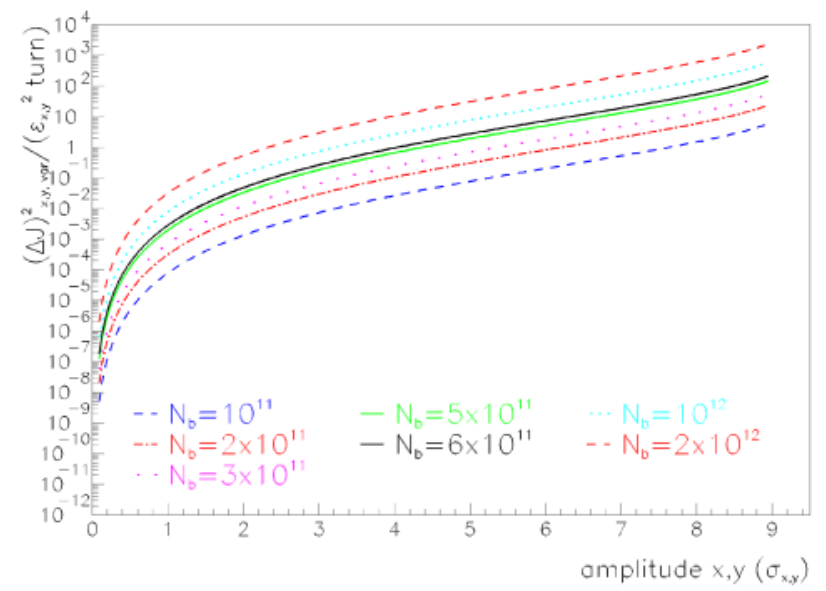

FIG. 14. (Color) Analytical estimate of the diffusion coefficient, Eq. (17), for different bunch populations.

\section{CONCLUSION}

Simulations show that the long-range beam-beam interaction may severely limit the dynamic aperture of future hadron colliders operating with many closely spaced bunches. Simulated threshold amplitudes for strong diffusion (diffusive aperture) are similar if either the exact expression for the beam-beam force or a $1 / r^{\prime}$ approximation is employed.

The Chirikov overlap criterion yields an analytical estimate for the onset of strong chaos. In order to apply the Chirikov criterion for determining the diffusive aperture caused by the long-range beam-beam interaction, we have derived analytical formulas for the tune shift with amplitude and the resonance driving terms, considering round beams and motion in the crossing plane only, through first-order perturbation theory.

Then restricting the treatment to the $1 / r^{\prime}$ part of the long-range beam-beam force, also taking into account the finite resonance width, and in addition assuming that a resonance is located exactly at the amplitude from which on resonance overlap can occur, we have derived an expression for the diffusive aperture. This analytical result, although slightly pessimistic, resembles the full simulation. We have demonstrated that the agreement with the latter can be improved further, by also accounting for the actual locations of resonances in phase space. In addition, we have estimated the magnitude of the diffusion coefficient in the chaotic region. For amplitudes larger than the diffusive aperture, the analytically estimated diffusion rate is in good agreement with the simulation.

Our analytical discussion clearly reveals the dependence of the long-range diffusive aperture on critical beam parameters, such as the crossing angle, the beta function at the IP, or the bunch population.

In much the same manner, resonant Hamiltonians could be computed for the full 4-dimensional transverse phase space, including alternating crossing at two interaction points. However, due to its purely geometrical character, 
extension of the overlap criterion to the topology of a higher-dimensional phase space is not possible.

\section{ACKNOWLEDGMENTS}

We would like to thank Y. Alexahin, O. Brüning, H. Burkhardt, J. Jowett, J.-P. Koutchouk, and A. Mostacci for useful discussions and suggestions. We are also grateful to W. Herr and F. Ruggiero for a careful reading of the manuscript, feedback, and corrections.

[1] L. R. Evans, in Proceedings of the CERN Accelerator School on Antiprotons in Colliding Beam Facilities, Geneva, Switzerland, 1984 (CERN Report No. CERN 84-15, 1984), p. 319.

[2] M. Meddahi, Ph.D. thesis, University de Paris VII, 1991 [CERN Report No. CERN SL/91-30 (BI), 1991].

[3] K. Cornelis, in Proceedings of the Workshop on Beam-Beam Effects in Large Hadron Colliders-LHC99, Geneva, 1999, edited by J. Poole and F. Zimmermann
(CERN Report No. CERN-SL-99-039 AP), 1999, p. 2; T. Satogata and S. Peggs, ibid., p. 108; F. Zimmermann, Ph.D. thesis, University of Hamburg, 1993.

[4] J. Irwin, Superconducting Super Collider Report No. 233, 1989 (unpublished).

[5] Y. Papaphilippou and F. Zimmermann, Phys. Rev. ST Accel. Beams 2, 104001 (1999).

[6] L. Leunissen, H. Grote, and F. Schmidt, in Proceedings of the EPAC 2000, Vienna, edited by M. Regler (European Physical Society, Geneva, 2000).

[7] J. Laskar, Astron. Astrophys. 198, 341 (1988); H. S. Dumas and J. Laskar, Phys. Rev. Lett. 70, 2975 (1993); J. Laskar, Physica (Amsterdam) 67D, 257 (1993); J. Laskar and D. Robin, Part. Accel. 54, 183 (1996).

[8] C. Polymilis, Ch. Skokos, G. Kollias, G. Servizi, and G. Turchetti, J. Phys. A 33, 1055 (2000).

[9] A. J. Lichtenberg and M. A. Lieberman, Regular and Chaotic Motion (Springer-Verlag, New York, 1992), p. 258.

[10] M. Abramowitz and I. A. Stegun, Handbook of Mathematical Functions (Dover, New York, 1972), p. 376.

[11] I. Gradshteyn and J. Ryzhik, Table of Integrals, Series, and Products (Academic Press, San Diego, 1994), 5th ed.

[12] B. V. Chirikov, Phys. Rep. 52, 5 (1979). 